\title{
miR-21 inhibitor facilitates the anticancer activity of doxorubicin loaded nanometer in melanoma
}

\author{
XIAOYONG WEI ${ }^{1,2}$, XIAOLONG YOU ${ }^{2}$, JIANLONG ZHANG ${ }^{2}$ and CUNCAI ZHOU ${ }^{2}$ \\ ${ }^{1}$ The Medical College of Nanchang University, Nanchang, Jiangxi 330006; \\ ${ }^{2}$ Department of Hepatobiliary Surgery, Jiangxi Cancer Hospital, Nanchang, Jiangxi 330029, P.R. China
}

Received June 5, 2018; Accepted May 13, 2019

DOI: $10.3892 /$ or.2019.7167

\begin{abstract}
MicroRNA-21 (miR-21) is a potential therapeutic target for melanoma. Whether miR-21 inhibitor affects the anti-cancer activity of doxorubicin assisted by $\mathrm{c}(\mathrm{RGDyK})$-modified liposome (DLN) in melanoma and the underlying mechanisms are largely unknown. In this study, in vitro and animal models were used to explore the effect of DLN combined with miR-21 inhibitor on melanoma cells. The data demonstrated that treatment with $5 \mu 1$ DLN (final concentration of doxorubicin $5 \mathrm{mg} / \mathrm{ml}$ ) for $72 \mathrm{~h}$ effectively inhibited melanoma cell growth ( $75 \%$ inhibition). The experiments were then divided into five groups: Control group, vector group, DLN group, miR-21 inhibitor group and miR-21 inhibitor + DLN group. Compared with the control group, DLN (5 $\mu \mathrm{l})$ or miR-21 inhibitor significantly reduced migration and invasion of melanoma cells, promoted apoptosis and arrested cells at the G1 phase. Notably, the combined application of DLN with miR-21 inhibitor further promoted the anti-cancer effects (reducing migration and invasion of melanoma cells, promoting apoptosis and arresting cells at G1 phase) compared with individual application of DLN or miR-21 inhibitor. Mechanically, DLN did not function by reducing miR-21 expression, whereas DLN and miR-21 inhibitor downregulated B-cell lymphoma-2 (BCL-2) expression, and facilitated BCL-2-associated X protein (Bax) and P53 expression in melanoma cells. DLN and miR-21 inhibitor together displayed stronger effects on Bcl-2, Bax and P53 expression that each alone. In vivo data further demonstrated that DLN inhibited tumor growth further than a similar dose of doxorubicin only. Furthermore, miR-21 inhibitor and DLN exerted the optimal anti-cancer effect compared with single application of DLN or miR-21 inhibitor. Together, the findings demonstrated miR-21 inhibitor facilitated the anti-cancer
\end{abstract}

Correspondence to: Dr Cuncai Zhou, Department of Hepatobiliary Surgery, Jiangxi Cancer Hospital, 519 Beijing East Road, Nanchang, Jiangxi 330029, P.R. China

E-mail: zhoucc719@sina.com

Key words: doxorubicin loaded nanometer, microRNA-21, melanoma, apoptosis activity of DLN in melanoma, and the mechanisms involved Bcl-2, Bax and P53 expression.

\section{Introduction}

Cancer is the second most common cause of mortality in the United States, and the incidence rate is still increasing (1). Chemotherapy is still the most common treatment among the four major cancer therapy types: Surgical treatment, radiotherapy, chemotherapy and biological therapy (2). The majority of the approaches currently used involve interference in nucleic acid metabolism and DNA repair (3). Currently, there are $>70$ types of anti-tumor drugs commonly used in the clinic, and $>400$ new anti-tumor drugs have entered clinical trials (4). However, the majority of drugs fail due to their lack of specificity for tumor cells.

Targeted therapy is a way of targeting tumor cells with drugs and other active substances that kill tumor cells without affecting the function of normal tissues and cells (5). Subsequently, the curative effect is improved and side effects are relatively reduced based upon the application of target therapy. The targeted drug delivery uses a novel nano drug delivery system, which increases the selectivity of the drug to the target focus, thus increasing the drug concentration in the tumor tissue (6,7). Current, the drug delivery systems mainlyact by targeting an antigen or antibody on the surface of tumor cells $(8,9)$. Doxorubicin is one of the common chemotherapeutic agents used to treat liver cancer (10). However, it has dose limitations due to its poor bioavailability, hydrophobicity and cytotoxicity. The cyclin-(RGDyk)[c(RGDyK)] peptide functionalized nanomicellar system can overcome the drawbacks of low transport of chemotherapeutics across the blood-tumor barrier (BTB) in multidrug-resistant cancer cells (11). The anti-tumor efficacy of chemotherapeutic agents was significantly facilitated with the assistance of the $\mathrm{c}(\mathrm{RGDyK}) \mathrm{drug}$ delivery system $(12,13)$. However, the therapeutic effects of doxorubicin assisted by $\mathrm{c}(\mathrm{RGDyK})$-modified liposomes on melanoma growth and the underlying mechanisms are largely unknown.

MicroRNAs (miRs) can act as oncogenes or tumor suppressor genes during cancer development $(14,15)$. miRs regulate multiple signaling pathways and change the expression of mRNAs $(16,17)$. miR-21 was reported to be upregulated in the tissues and cells of various solid tumors, including 
glioma, colon cancer, gastric cancer and melanoma tissue (18). Overexpression of miR-21 in glioma cells can inhibit apoptosis and miR-21 may have an oncogenic function (19). miR-21 is a potential therapeutic target for various types of cancer, particularly melanoma (18). Therefore, a miR-21 inhibitor could potentially facilitate the antitumor effect of a targeted therapy. In this study, cellular and animal models were used to explore the effect of doxorubicin capsuled in 4c[RGDyk]-L-[CD] nanoparticle and miR-21 inhibitor on melanoma cells, and to assess its associated mechanisms. This study may provide an experimental basis for the treatment of melanoma.

\section{Materials and methods}

Preparation of doxorubicin-loaded nanometer. Doxorubicin was obtained from Beijing Huafeng United Technology Co., Ltd. (Beijing, China). c(RGDyK) (molecular weight, 619.51) was synthesized by GL Biochem Ltd. (Shanghai, China). Liposomes loaded with doxorubicin and c(RGDyK)-L-[CD] were prepared in our lab using the method described previously (20). Doxorubicin (1 ml) diluted in PBS was added into a dialysis bag. After $48 \mathrm{~h}$, the dialysis bag was cut and $2 \mathrm{ml} \mathrm{10 \%}$ Triton X-100 was added to destroy all the liposomes. The final concentration of doxorubicin was $20 \mathrm{mg} / \mathrm{ml}$.

Cell culture. Melanoma cell line B16F10 was purchased from Shanghai Cell Bank of Chinese Academy of Sciences and cultured in Dulbecco's modified Eagle's medium (DMEM; Gibco; Thermo Fisher Scientific, Inc., Waltham, MA, USA) supplemented with $10 \%$ fetal bovine serum (FBS; HyClone; GE Healthcare Life Sciences, Logan, UT, USA) and $100 \mathrm{U} / \mathrm{ml}$ penicillin-streptomycin (Sigma-Aldrich; Merck $\mathrm{KGaA}$, Darmstadt, Germany) in $5 \% \mathrm{CO}_{2}$ at $37^{\circ} \mathrm{C}$. When the cell confluence reached $90 \%$, the culture medium was discarded and cells were washed twice with PBS and passaged following trypsin digestion.

miR-21 inhibitor transfection. At a density of 50-70\%, B16F10 cells $\left(3 \times 10^{3}\right.$ cells) were transfected with vector (miR-21 scrambled control) or inhibitor (1 $\mu \mathrm{l})$ using Lipofectamine ${ }^{\mathrm{TM}} 2000$ (Thermo Fisher Scientific, Inc.) according to the manufacturer's instructions for $6 \mathrm{~h}$. The miR-21 inhibitor and miR-21 negative control (NC) inhibitor were synthesized by Sangon Biotech Co., Ltd. (Shanghai, China). Cells were then cultured in DMEM containing $10 \%$ FBS for another $72 \mathrm{~h}$. The sequences of miR-21 inhibitor and vector were 5'-UCAACAUCAGUC UGAUAAGCUA-3' and 5'-UCUACUCUUUCUAGGAGG UUGUGA-3'.

MTT assay. The effects of doxorubicin-loaded nanometer (DLN) on melanoma cell growth were evaluated by MTT assay. The cells were treated with different concentrations of DLN $(0-20 \mu \mathrm{l})$ for 24-72 $\mathrm{h}$. To investigate the anti-tumor effects of association application of DLN with miR-21 inhibitor, the experiments were divided into five groups: Control group, vector (miR-21 inhibitor NC) group, DLN group, miR-21 inhibitor group, and miR-21 inhibitor + DLN group. Following the indicated treatments, an MTT assay was applied to evaluate cell proliferation. DMSO $(100 \mu \mathrm{l})$ was added to each well to dissolve the formazan. The optical density was determined via microplate reader (BioTek Instruments, Inc., Winooski, VT, USA) at $570 \mathrm{~nm}$ and represented cell viability.

Transwell assay. B16F10 cells in the logarithmic growth phase were cultured in serum-free medium for $24 \mathrm{~h}$. The medium was discarded and the cells were digested using trypsin. The cells underwent washing, centrifugation and resuspension in a low serum DMEM medium (containing $0.2 \% \mathrm{FBS}$ ) to form a single cell suspension with a density of $5 \times 10^{5} / \mathrm{ml}$. The cells in different groups were treated for $48 \mathrm{~h}$. Subsequently, the cells were collected and suspended cells $\left(100 \mu \mathrm{l} ; 1 \mathrm{x} 10^{6} / \mathrm{ml}\right)$ were added into each Transwell chamber. DMEM/F12 (600 $\mu \mathrm{l})$ containing $10 \%$ FBS was added into each well in a 24 -well plate. The prepared Transwell chambers were placed in the 24-well plate for $20 \mathrm{~h}$ at $37^{\circ} \mathrm{C}$ and $5 \% \mathrm{CO}_{2}$. Cells in the lower chamber were fixed in $4 \%$ paraformaldehyde for $30 \mathrm{~min}$ at room temperature and stained with $3 \%$ crystal violet for $5 \mathrm{~min}$ at room temperature. Four fields in the upper and lower, left and right positions were selected for counting in each well. The experiment was repeated for three times and mean value was calculated.

Cell migration. B16F10 cells $\left(3 \times 10^{5}\right)$ were seeded in 6-well plates and treated with the indicated drugs for $72 \mathrm{~h}$. After that, the cells were collected and suspended cells (100 $\mu \mathrm{l}$; $1 \times 10^{6} / \mathrm{ml}$ ) were incubated in the plates for $24 \mathrm{~h}$. A line was drawn across the center of the wells. After incubation in $\mathrm{CO}_{2}$ incubator at $37^{\circ} \mathrm{C}$, the images were captured under a light microscope. The migration speed was calculated based on the formula: Cell mobility $(\mu \mathrm{m} / \mathrm{h})=\left[\right.$ width $_{(1)}-$ width $\left._{(2)}\right] / 72 \mathrm{~h}$. Width $_{(1)}$ and width ${ }_{(2)}$ represented the width measured at 0 and $72 \mathrm{~h}$, respectively.

Flow cytometry. B16F10 cells $\left(3 \times 10^{5}\right)$ were seeded in 6-well plates and treated with indicated drugs for $72 \mathrm{~h}$. The cells were collected following trypsin digestion. The cells were incubated with Annexin V-fluorescein isothiocyanate $(0.5 \mu \mathrm{l})$ and propidium iodide (PI; $0.5 \mu \mathrm{l}$; cat. no. C1062; Beyotime Institute of Biotechnology, Haimen, China) for $30 \mathrm{~min}$ in the dark. Subsequently, apoptosis was detected by flow cytometry (BD Biosciences, San Jose, CA, USA) and data were analyzed using FlowJo 10 (FlowJo LLC, Ashland, OR, USA). Following transfection for $72 \mathrm{~h}$, the cells were collected for PI staining $(0.5 \mu \mathrm{l} / \mathrm{ml})$ and the cell cycle distribution was assessed by flow cytometry (BD Biosciences) within $1 \mathrm{~h}$ of staining.

Reverse transcription-quantitative polymerase chain reaction $(R T-q P C R)$. B16F10 cells $\left(3 \times 10^{5}\right)$ were seeded in 6-well plates and treated with indicated drugs for $72 \mathrm{~h}$. RNA in different groups was extracted using a TRIzol (Thermo Fisher Scientific, Inc.). Subsequently, RNA was transcribed into cDNA according to the instructions of a Reverse Transcription Kit (Takara Biotechnology Co., Ltd., Dalian, China). Fluorescence qPCR was utilized to detect the expression level of the target RNAs using cDNA as the template following the instruction of Platinum ${ }^{\mathrm{TM}}$ II Taq Hot-Start DNA Polymerase (cat. no. 14966005; Thermo Fisher Scientific, Inc.). SYBR-Green (cat. no. HY-K0501; MedChemExpress, Monmouth Junction, NJ, USA) was used as fluorophore and the thermocycling 
Table I. Primer sequence for use in reverse transcription-quantitative polymerase chain reaction.

\begin{tabular}{|c|c|c|c|c|}
\hline Primer name & Sequence $\left(5^{\prime}-3^{\prime}\right)$ & Primer length (bp) & Product length (bp) & Annealing $\left({ }^{\circ} \mathrm{C}\right)$ \\
\hline $\operatorname{miR}-21 \mathrm{~F}$ & CGCCGTAGCTTATCAGACTG & 20 & 65 & 57.7 \\
\hline $\operatorname{miR}-21 \mathrm{R}$ & CAGCCACAAAAGAGCACAAT & 20 & & \\
\hline U6 F & CTCGCTTCGGCAGCACA & 17 & 94 & 60 \\
\hline U6 R & AACGCTTCACGAATTTGCGT & 20 & & \\
\hline BCL-2 F & GTGCCTGCTTTTAGGAGACCGA & 22 & 128 & 62.9 \\
\hline BCL-2 R & GAGACCACACTGCCCTGTTGATC & 23 & & \\
\hline P53 F & AGTGCTCGCTTAGTGCTCCCT & 21 & 110 & 62.6 \\
\hline P53 R & GTGCATGTTTGTGCCTGTCCT & 21 & & \\
\hline Bax F & AGACACTCGCTCAGCTTCTTG & 21 & 116 & 58 \\
\hline Bax R & CTTTTGCTTCAGGGTTTCATC & 21 & & \\
\hline GAPDH F & GAAGGTCGGAGTCAACGGAT & 20 & 224 & 58.3 \\
\hline GAPDH R & CCTGGAAGATGGTGATGGG & 19 & & \\
\hline
\end{tabular}

miR-21, microRNA-21; F, forward; R, reverse; BCL-2, B-cell lymphoma-2; Bax, BCL-2-associated X protein.

conditions were as follows: Initial denaturation at $95^{\circ} \mathrm{C}$ for $10 \mathrm{~min}$, followed by 40 cycles of a two-step PCR at $95^{\circ} \mathrm{C}$ for $15 \mathrm{sec}$ and $60^{\circ} \mathrm{C}$ for $1 \mathrm{~min}$. The expression of miR-21 was normalized to U6. The expression of BCL-2, Bax and P53 was normalized to GAPDH. The primers are listed in Table I. The $2^{-\Delta \Delta \mathrm{Cq}}$ method was used to quantify the results as previously described (21).

Western blot. B16F10 cells $\left(3 \times 10^{5}\right)$ were seeded in 6-well plates and treated with indicated drugs for $72 \mathrm{~h}$. Protein was extracted from the treated cells using a protein isolation kit (cat. no. 28-9425-44, GE Healthcare Life Sciences). Protein levels were quantified with a bicinchoninic acid protein assay kit. Protein $(25 \mu \mathrm{g} /$ lane) was separated via SDS-PAGE on $12 \%$ gels and transferred onto nitrocellulose membranes. The membranes were blocked in 5\% skim milk for $2 \mathrm{~h}$ at room temperature. The antibodies against BCL-2 (cat. no. ab32124; Abcam, Cambridge, MA, USA; 1:1,000), Bax (cat. no. ab32503; Abcam; 1:2,000), P53 (cat. no. A11212; ABclonal Biotech Co., Ltd., Woburn, MA, USA; 1:1,000) and GAPDH (cat. no. AC033; ABclonal Biotech Co., Ltd.; $1: 1,000)$ were incubated overnight at $4^{\circ} \mathrm{C}$. After washing, the membranes were incubated with the secondary antibody (horseradish peroxidase-labeled goat anti-rabbit $\operatorname{IgG} ; 1: 100$; cat. no. ab131368; Abcam) for $2 \mathrm{~h}$ at room temperature. Enhanced chemiluminescence kit (cat. no. RPN2133; GE Healthcare Life Sciences) was added to the membrane and visualized using a gel imaging system (Bio-Rad Laboratories, Inc., Hercules, CA, USA).

Establishment of in vivo tumor model. All animal experiments were approved by the Ethics Committee of Jiangxi Tumor Hospital (approval no. 20170312). Female Balb/c nude mice (6-week old; $20 \mathrm{~g}$ ) were purchased from Hunan SLAC Experimental Animal Co. Ltd. (SCXK2016-0002) and housed in a specific pathogen-free condition that was automatically maintained at a temperature of $23 \pm 2^{\circ} \mathrm{C}$, a relative humidity of $45-65 \%$, and with a controlled $12 \mathrm{~h}$ light/dark cycle. The mice implanted with the tumor cell lines were randomly divided into six groups ( $\mathrm{n}=5$ in each group): Control group, doxorubicin group, DLN group, miR-21 inhibitor group, miR-21 inhibitor + doxorubicin group and miR-21 inhibitor + DLN group. B16F10 cells in the logarithmic growth phase $\left(1 \times 10^{7}\right)$ were diluted in $0.2 \mathrm{ml}$ PBS and injected (subcutaneous injection) into the Balb/c nude mice. Doxorubicin, DLN and miR-21 inhibitor group were administered for 10 consecutive days, following attainment of a tumor size of $50 \mathrm{~mm}^{3}$. In the miR-21 group, each mouse received miR-21 inhibitor [intraperitoneal injection (i.p.), $20 \mu \mathrm{l}]$. In the miR-21 inhibitor + doxorubicin group, each mouse received $20 \mu \mathrm{l}(1 \mu \mathrm{g} / \mu \mathrm{l}) \mathrm{miR}-21$ inhibitor $(i . p$.$) and$ $20 \mu \mathrm{l}$ doxorubicin $(5 \mathrm{mg} / \mathrm{kg})$. In the miR-21 inhibitor + DLN group, each mouse received $20 \mu \mathrm{lmiR}-21$ inhibitor (i.p.) and $20 \mu \mathrm{l}$ DLN $(5 \mathrm{mg} / \mathrm{kg})$. General conditions of the mice were monitored daily and the tumor size was measured every 2 days. Following 10 days of drug administration, the mice were anesthetized using isoflurane and decapitated, and the whole tumor was removed. The tumor specimens were fixed in $4 \%$ paraformaldehyde in $\mathrm{PBS}$ ( $\mathrm{pH} \mathrm{7.4)}$ ) at $4^{\circ} \mathrm{C}$ overnight and then embedded in paraffin for tissue sectioning. The tissues were sectioned into $5 \mu \mathrm{m}$-thick sections. Subsequently, the slides were stained with hematoxylin $(3 \%)$ and eosin $(3 \%)$ for $5 \mathrm{~min}$ at room temperature. The images were captured under light microscopy.

Statistical analysis. The data were presented as the mean + standard deviation and analyzed using SPSS 17.0 (SPSS, Inc., Chicago, IL, USA). Statistical significance was determined by one-way analysis of variance with Newman-Keuls as the post-hoc test. $\mathrm{P}<0.05$ was considered to indicate a statistically significant difference.

\section{Results}

DLN inhibits cell growth of B16F10 cells. Initially, we detected the effects of $5 \mu \mathrm{l}$ DLN on cell growth of B16F10 cells. As shown in Fig. 1A, DLN inhibits the cell growth in a 

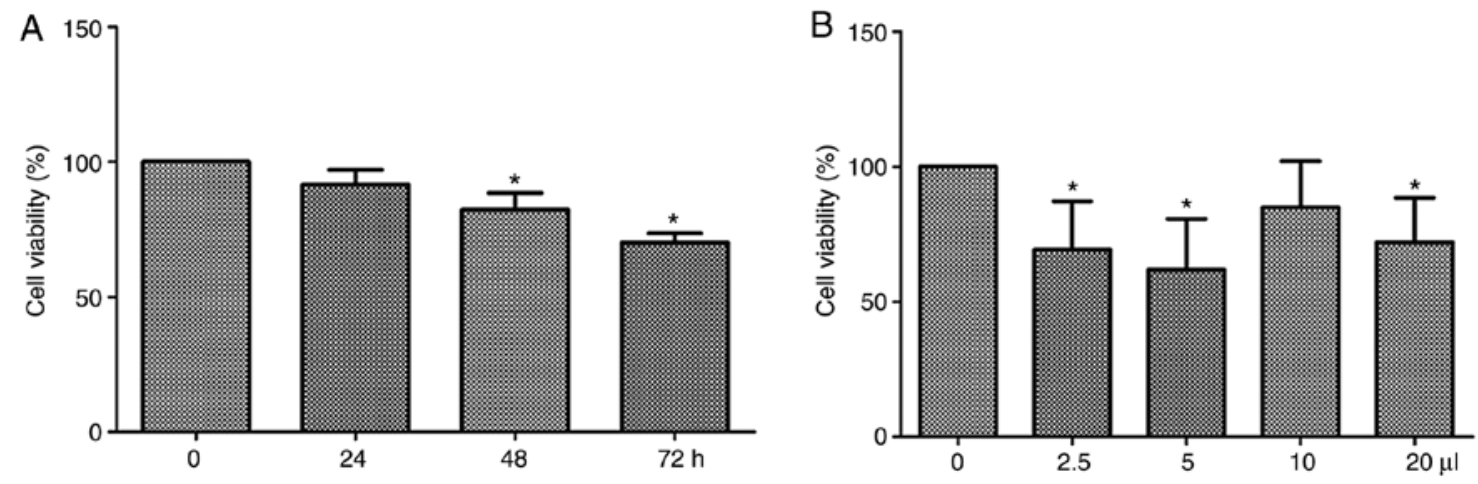

Figure 1. DLN inhibits cell growth of B16F10 cells. (A) DLN inhibits the cell growth in a time-dependent manner (0-72 h); (B) DLN with $5 \mathrm{mg} / \mathrm{ml} \mathrm{doxorubicin}$ exhibited the optimal effects on cell growth after $72 \mathrm{~h}$ treatment. ${ }^{*} \mathrm{P}<0.05$ vs. control. DLN, doxorubicin-loaded nanometer.

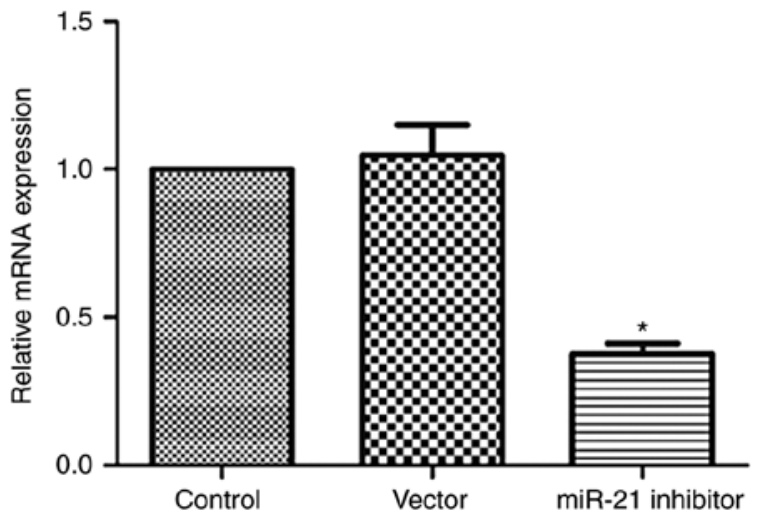

Figure 2. miR-21 inhibitor reduces miR-21 expression. miR-21 inhibitor reduced miR-21 expression, while control vector did not affect miR-21 expression. ${ }^{*} \mathrm{P}<0.05$ vs. control. miR-21, microRNA-21.

time-dependent manner (0-72 h). Significant difference was observed at the 48 and $72 \mathrm{~h}$ time points compared with the $0 \mathrm{~h}$ control $(\mathrm{P}<0.05)$. As shown in Fig. 1B, $5 \mu$ l DLN $(5 \mathrm{mg} / \mathrm{ml})$ the optimal effects on cell growth were observed after $72 \mathrm{~h}$ treatment. Therefore, $5 \mu \mathrm{l}$ DLN was selected to treat the cells for $72 \mathrm{~h}$ in the subsequent experiments.

miR-21 inhibitor reduces miR-21 expression. miR-21 expression was confirmed by RT-qPCR. As shown in Fig. 2, miR-21 inhibitor significantly reduced miR-21 expression $(\mathrm{P}<0.05)$, while control miR-21 inhibitor NC did not affect miR-21 expression.

miR-21 inhibitor facilitates the anti-migration effect of DLN. As shown in Fig. 3, DLN significantly inhibited the migration of melanoma cells $(\mathrm{P}<0.05)$. miR-21 inhibitor also significantly inhibited cell migration compared with control $(\mathrm{P}<0.05)$. Combined application of DLN and miR-21 inhibitor further inhibited cell migration compared with single application of DLN and miR-21 inhibitor.

miR-21 inhibitor facilitates the anti-invasion effect of DLN. As shown in Fig. 4, DLN significantly inhibited invasion of melanoma cells at the concentration of $5 \mu \mathrm{l}(\mathrm{P}<0.05)$. miR-21 inhibitor also significantly inhibited cell invasion $(\mathrm{P}<0.05)$. Results demonstrated that DLN and miR-21 inhibitor combination further inhibited cell invasion compared with DLN or miR-21 inhibitor alone.

miR-21 inhibitor promotes cell cycle arrest following DLN treatment. As shown in Fig. 5, miR-21 inhibitor and DLN increased the number of cells at G1 phase, and decreased the number of cells at $\mathrm{S}$ phase $(\mathrm{P}<0.05)$. miR-21 inhibitor further increased cell cycle arrest ability of DLN when combined treatment was performed.

miR-21 inhibitor facilitates the apoptosis induced by DLN. As shown in Fig. 6, DLN induced apoptosis of melanoma cells at a concentration of $5 \mu \mathrm{l}(\mathrm{P}<0.05)$. miR-21 inhibitor also induced apoptosis of melanoma cells $(\mathrm{P}<0.05)$. The co-application of miR-21 inhibitor and DLN produced a further increase in apoptosis compared with single application of DLN and miR-21 inhibitor.

miR-21 inhibitor promotes the effects of DLN on BCL-2, P53 and Bax. miR-21 and apoptosis-related gene expression were also detected. As shown in Fig. 7A, miR-21 expression in miR-21 inhibitor group and miR-21 inhibitor + DLN group was significantly reduced compared with control $(\mathrm{P}<0.05)$. By contrast, DLN treatment alone did not affect miR-21 expression. BCL-2 mRNA expression was downregulated by miR-21 inhibitor or DLN treatment $(\mathrm{P}<0.05)$. Additionally, the associated application of miR-21 inhibitor with DLN further reduced BCL-2 expression. Bax mRNA expression was upregulated by miR-21 inhibitor or DLN treatment $(\mathrm{P}<0.05)$. Furthermore, the associated application of miR-21 inhibitor with DLN further promoted Bax expression. P53 mRNA expression was upregulated by miR-21 inhibitor or DLN treatment $(\mathrm{P}<0.05)$, and the combined application of miR-21 inhibitor with DLN further promoted P53 expression. The protein expression of BCL-2, Bax and P53 was confirmed by western blotting, with a similar trend observed in different groups as the mRNA expression (Fig. 7B and C).

miR-21 inhibitor facilitates the anti-tumor effects of $D L N$ in vivo. Mouse body temperature and behaviors were monitored each day. In the present study, the mice displayed normal wellbeing and all animals presented with a single subcutaneous tumor in the forelimb armpit and the longest diameter was $<10 \mathrm{~mm}$. Furthermore, no 

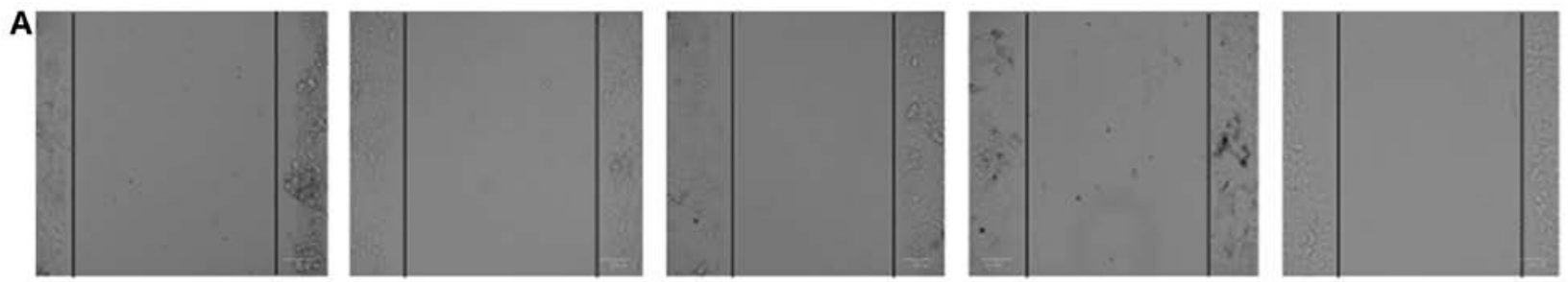

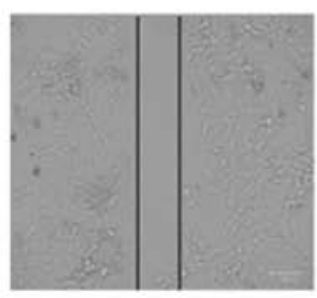

Control

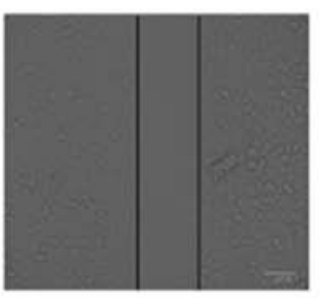

Vector

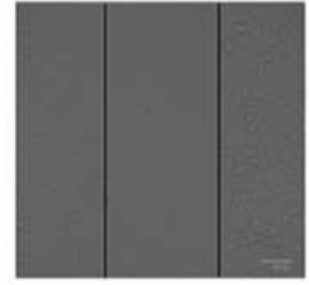

Nanometer

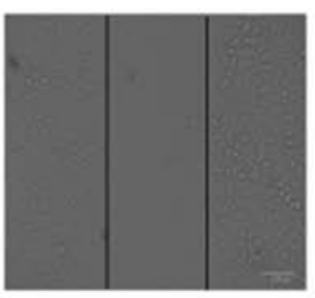

miR-21 inhibitor

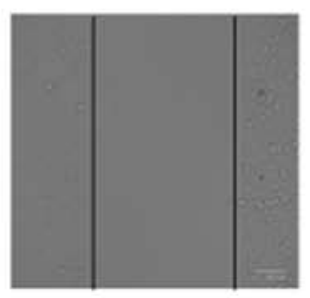

miR-21 inhibitor+ Nanometer

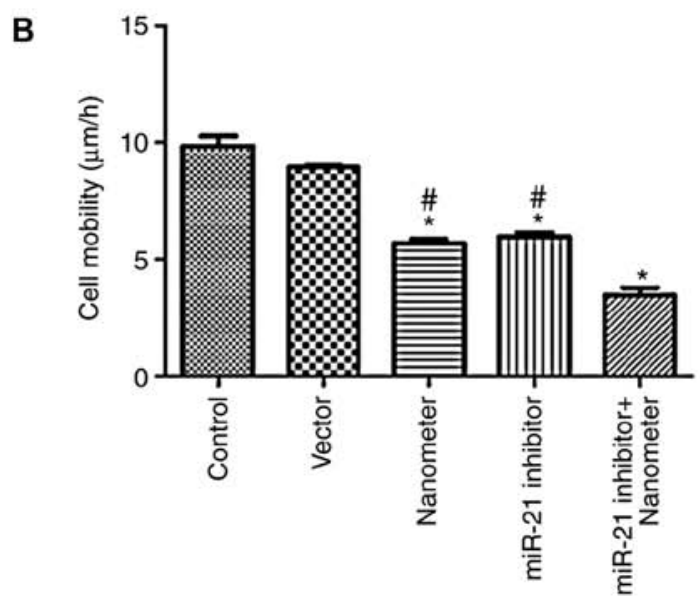

Figure 3. miR-21 inhibitor facilitates the anti-migration effect of DLN. (A) Representative images at 0 and $72 \mathrm{~h}$. (B) Quantification of migrated cells. Results are presented as the mean \pm standard error of six replicates. ${ }^{*} \mathrm{P}<0.05$ vs. control; ${ }^{*} \mathrm{P}<0.05$ vs. miR-21 inhibitor + DLN. DLN, doxorubicin-loaded nanometer; miR-21, microRNA-21.

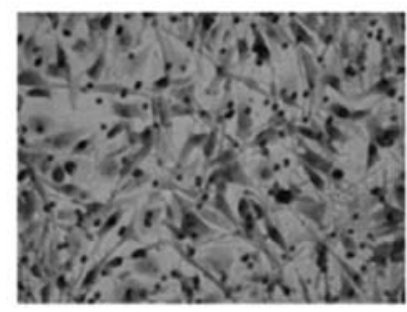

Control

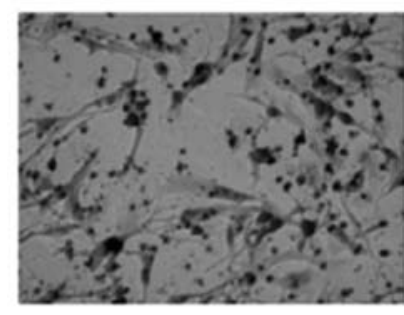

miR-21 inhibitor

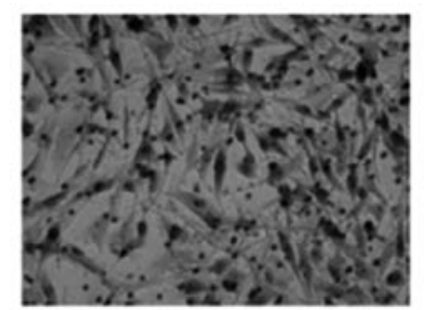

Vector

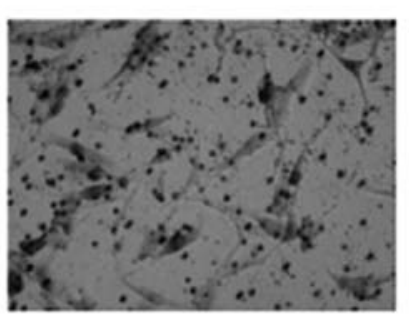

miR-21 inhibitor+ Nanometer

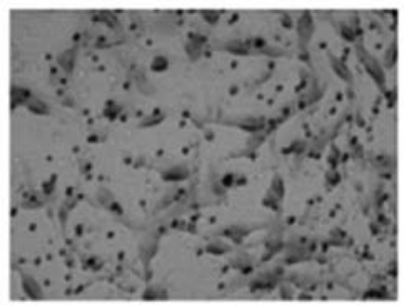

Nanometer

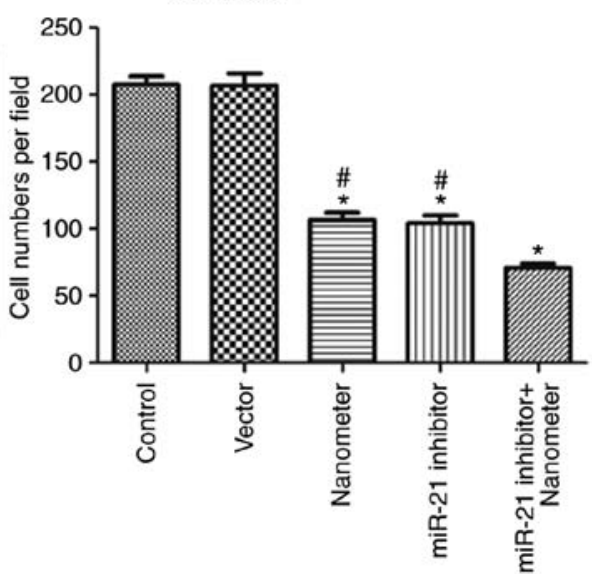

Figure 4. miR-21 inhibitor facilitates the anti-invasion effect of DLN. DLN significantly inhibited invasion of melanoma cells at the concentration of $5 \mu 1$. miR-21 inhibitor also inhibited cell invasion. DLN and miR-21 inhibitor combination further inhibited cell invasion. ${ }^{*} \mathrm{P}<0.05$ vs. control; ${ }^{\#} \mathrm{P}<0.05$ vs. miR-21 inhibitor + DLN. DLN, doxorubicin-loaded nanometer; miR-21, microRNA-21. 

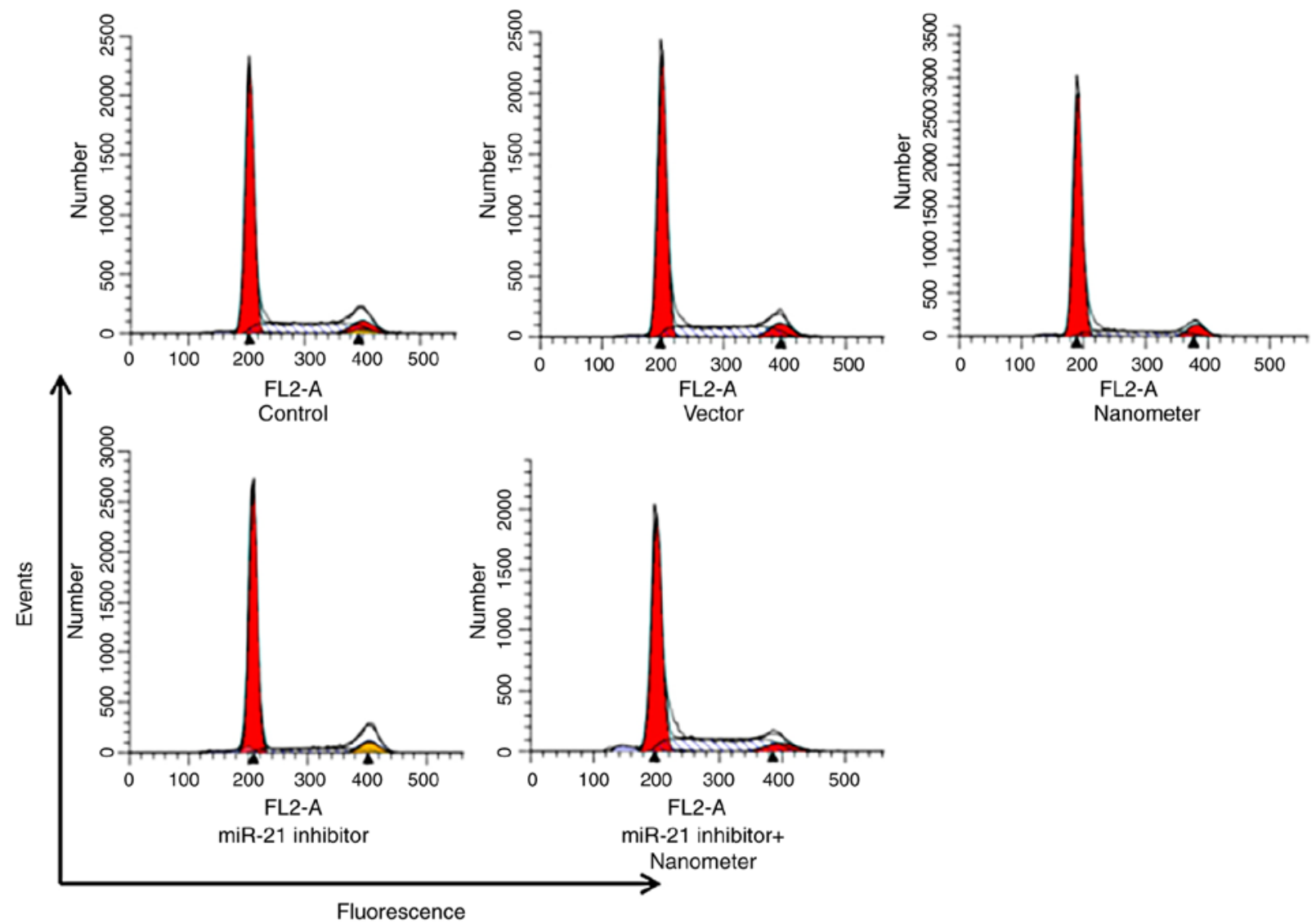

Fluorescence

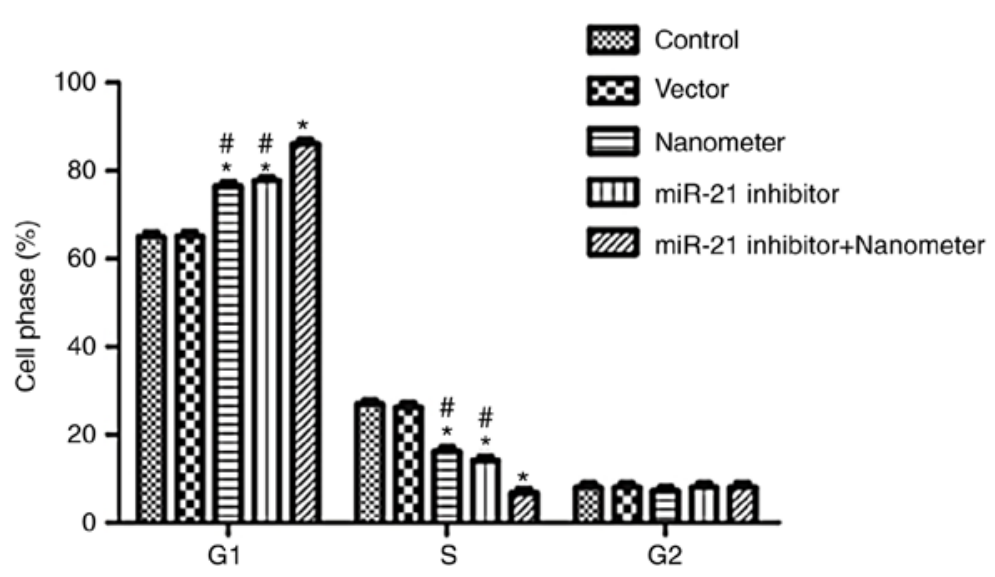

Figure 5. miR-21 inhibitor increases cell cycle arrest of DLN. Upper panel: Representative images of flow cytometry and quantification data of cell cycle distribution. " $\mathrm{P}<0.05$ vs. control; ${ }^{*} \mathrm{P}<0.05$ vs. miR-21 inhibitor + DLN. DLN, doxorubicin-loaded nanometer; miR-21, microRNA-21.

ulceration or abrasion of tumor surface was observed. The maximum tumor burden was observed in the control group ( $\sim 8 \%$ of the body weight). As shown in Fig. 8, tumor weight in the doxorubicin, DLN, miR-21 inhibitor groups were significantly reduced compared with control $(\mathrm{P}<0.05)$. The combined treatment of miR-21 inhibitor with DLN further inhibited tumor growth compared with individual of miR-21 inhibitor or DLN treatment. As shown in Fig. 9, the tumor tissue in control group was rich in blood vessels. The tumor cells were arranged closely and the size was relatively consistent. Tumor cells in the doxorubicin group were loosely arranged, and tumor cells in the DLN group were significantly enlarged. Tumor cells in miR-21 inhibitor group exhibited vacuoles and necrosis. More tumor cells in miR-21 inhibitor + doxorubicin group exhibited necrosis. In addition, tumor cells in miR-21 inhibitor + DLN group were loosely arranged.

\section{Discussion}

In this study, in vitro cellular and animal models were used to explore the effect of DLN combined with miR-21 inhibitor on melanoma tumor cells. Our data revealed that DLN had stronger anticancer effects compared with direct application of doxorubicin. From a mechanistic perspective, DLN did not affect miR-21 expression to exert its antitumor effects, which was facilitated by miR-21 inhibitor. These data provided novel idea for the application of DLN in melanoma treatment.

Melanoma is a skin cancer caused by the dedifferentiation of melanocytes (22). Melanoma is characterized by 

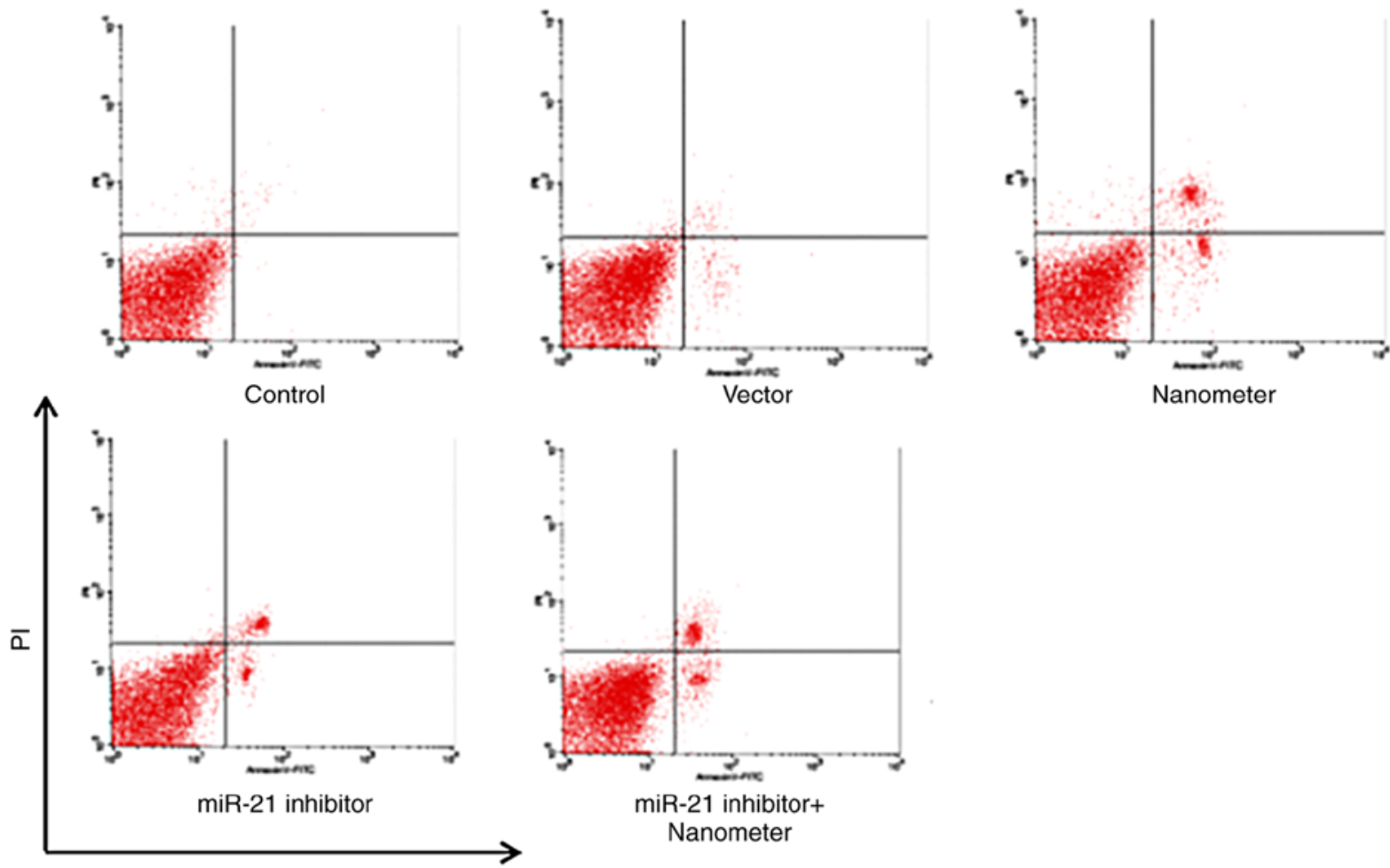

Annexin V-FITC

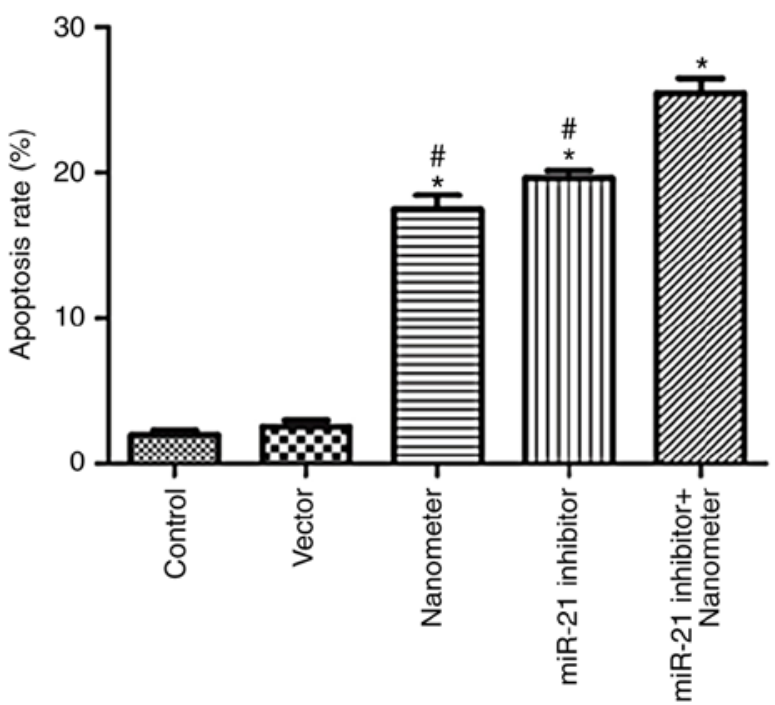

Figure 6. miR-21 inhibitor facilitates the apoptosis induced by DLN. Upper panel: Representative images of flow cytometry. Lower panel: Quantification data of apoptosis. ${ }^{*} \mathrm{P}<0.05$ vs. control; ${ }^{*} \mathrm{P}<0.05$ vs. miR-21 inhibitor + DLN. DLN, doxorubicin-loaded nanometer; miR-21, microRNA-21.

strong immune escape ability (23). Currently, the treatment of melanoma is typically early surgery, followed by chemotherapy combined with biological therapy. As malignant tumor is caused by unlimited proliferation and abnormal differentiation (24), the available treatments involve stimulating differentiation and apoptosis. However, traditional chemotherapeutic drugs still have various problems. The most prominent is that chemotherapy drugs not only kill tumor cells, but also selectively damage normal cells, particularly hematopoietic tissue and cells (25). The C[RGDyk]-modified liposome targeted delivery system is one of the common active tumor-targeting drug delivery systems (26). This system improves therapeutic efficacy in bone metastasis from breast cancer (27) and glioblastoma (28). In this study, the anticancer effect of DLN in melanoma was investigated.

MTT assay was used to determine the appropriate concentration and appropriate time for the administration of the drug delivery system, which provided the basis for the following experiments. Based upon the data, $5 \mu \mathrm{l}$ DLN $(5 \mathrm{mg} / \mathrm{ml})$ was selected and the cells were treated for $72 \mathrm{~h}$. In the current study, the effect of $10 \mu 1$ DLN on cell proliferation was not observable. Although the reasons were not identified, high concentrations of C[RGDyk]-modified liposome may affect the bioavailability of doxorubicin. These data receive more attention in a future study. miR-21 is a potential therapeutic target in melanoma $(18,29,30)$. In this present study, miR-21 inhibitor was applied in melanoma cells. The results confirmed 

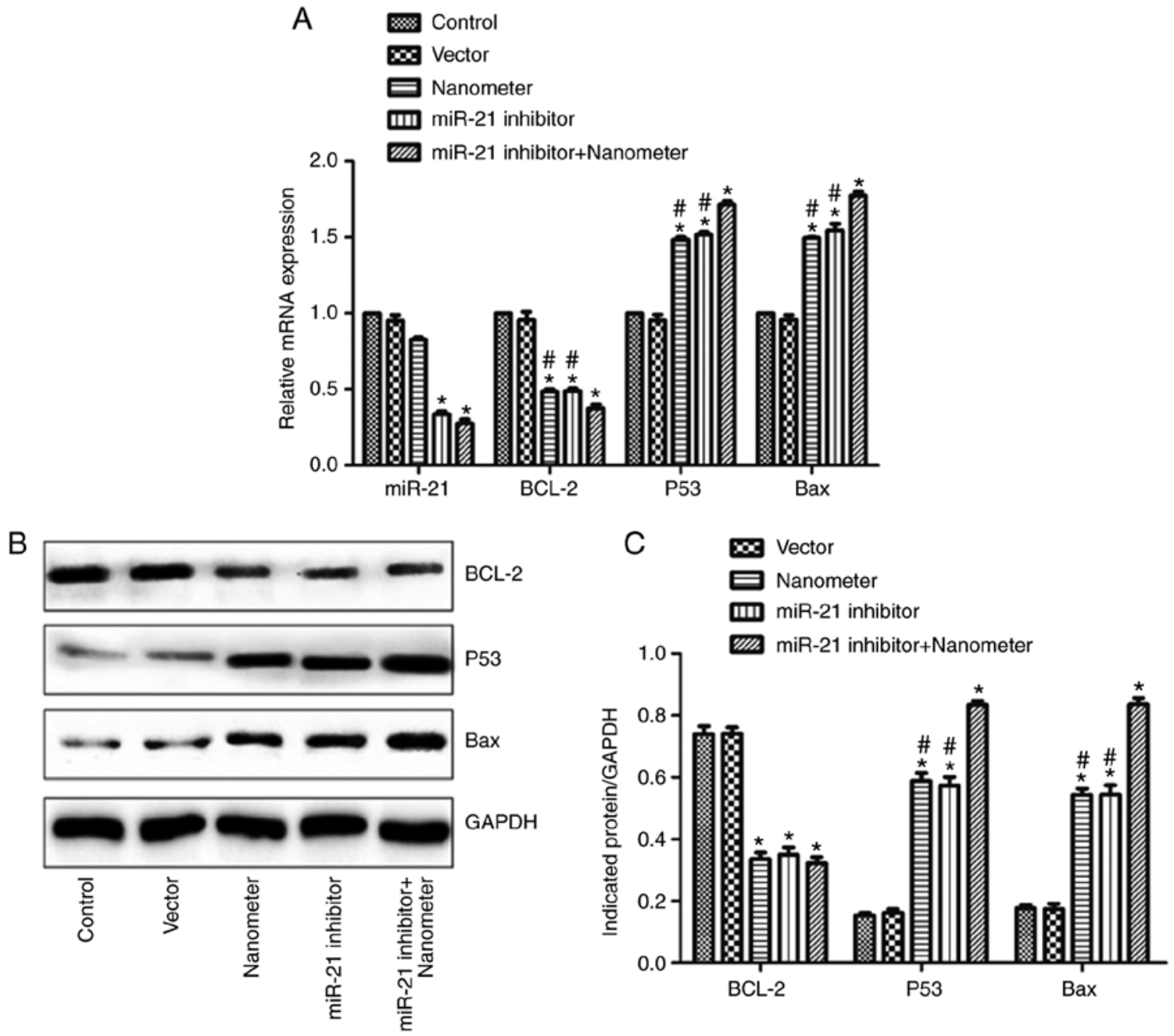

Figure 7. miR-21 inhibitor promotes the effects of on BCL-2, P53 and Bax. (A) miR-21 expression and mRNA expression of BCL-2, Bax and P53; (B) Protein expression of BCL-2, Bax and P53 was analyzed by western blot and (C) semi-quantified. The associated application of miR-21 inhibitor with DLN further promoted Bax and P53 expression, and reduced BCL-2 expression. ${ }^{*} \mathrm{P}<0.05$ vs. Control; ${ }^{*} \mathrm{P}<0.05$ vs. miR-21 inhibitor + DLN. DLN, doxorubicin-loaded nanometer; BCL-2, B-cell lymphoma-2; Bax, BCL-2-associated X protein; miR-21, microRNA-21.

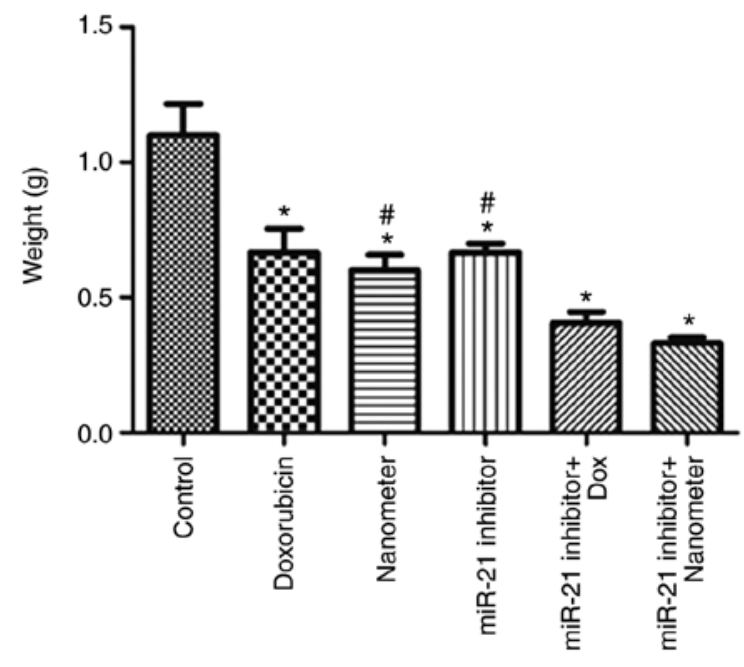

Figure 8. miR-21 inhibitor facilitates the anti-tumor effects of in vivo. Tumor weight in doxorubicin, DLN, miR-21 inhibitor groups were significantly reduced compared with control. The associated application of miR-21 inhibitor with DLN further inhibited tumor growth compared with single application of miR-21 inhibitor or DLN. "P<0.05 vs. Control; ${ }^{\text {"}} \mathrm{P}<0.05$ vs. miR-21 inhibitor + DLN. DLN, doxorubicin-loaded nanometer miR-21, microRNA-21. that the miR-21 inhibitor significantly reduced the expression of miR-21 in melanoma cells. More importantly, miR-21 inhibitor further promoted the anti-tumor effects of DLN, including inhibiting cell proliferation, invasion and migration, and promoting apoptosis of melanoma cells. In this study, the reduced proliferation following treatment with DLN may affect the cell invasion and migration results. Therefore, we resuspended the cells after treatments and similar numbers of cells in different groups were used in the analysis of cell migration and invasion, which may alleviate the bias of migration and invasion caused by reduced cell proliferation.

Invasive melanomas are characterized by the growth of melanocyte nests, which are surrounded by interstitial matrix and various types of stromal and immune cells. The surrounding tissue is typically matrix rich (31). Integrins are heterodimeric, transmembrane receptors that function as mechanosensors, adhesion molecules and signal transduction platforms in a multitude of biological processes, particularly in tumorigenesis (32). Self-aggregated pegylated poly-nanoparticles were tagged with $\mathrm{c}(\mathrm{RGDyK})$ peptide for targeted doxorubicin delivery to integrin-rich tumors (33). This may relieve the side effects of chemotherapeutics on normal organs. 


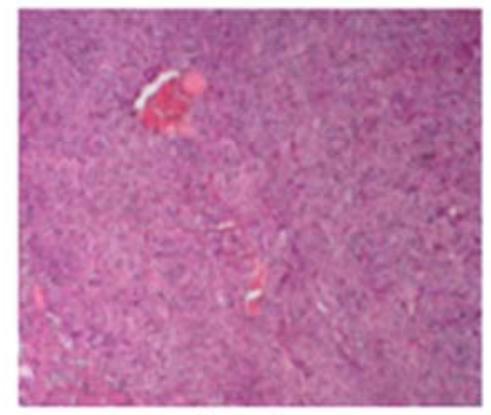

Control

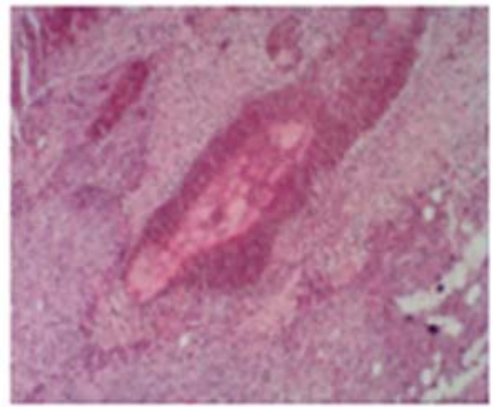

miR-21 inhibitor

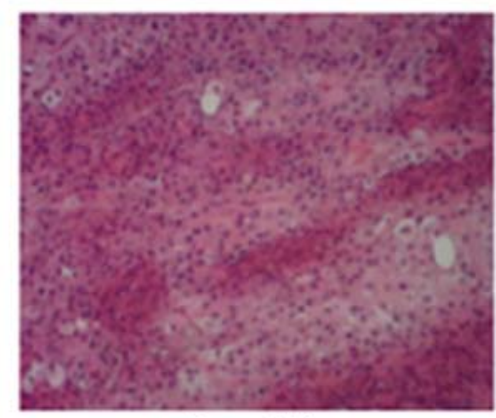

Doxorubicin

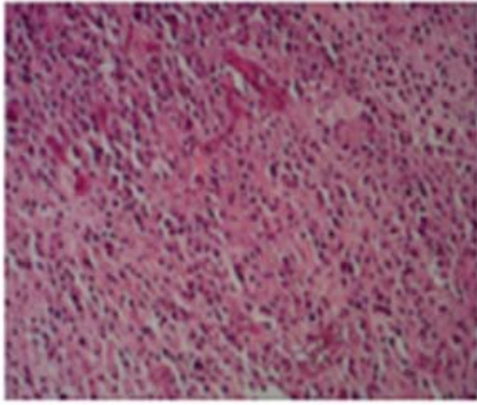

miR-21 inhibitor+Dox

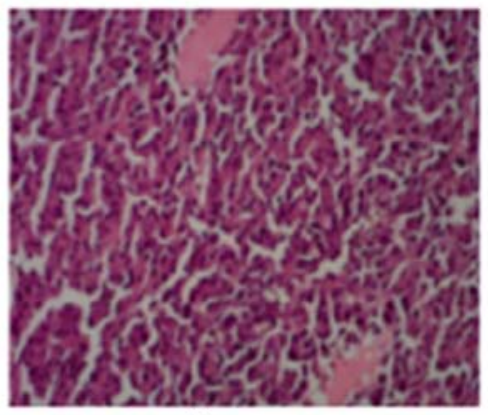

Nanometer

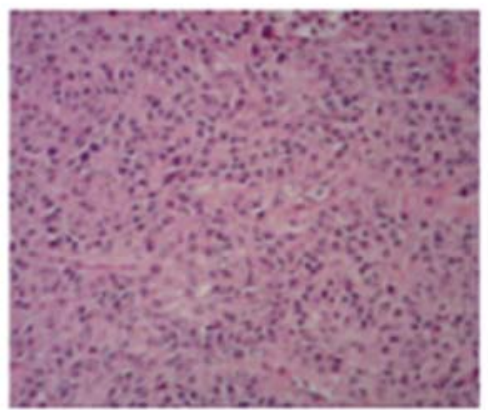

miR-21 inhibitor+Nanometer

Figure 9. Hematoxylin and eosin staining of the tumor tissues (x200). The tumor tissue in control group was rich in blood vessels. The tumor cells were arranged closely and the size was relatively consistent. By contrast, the tumor cells in other groups were loosely arranged and the cell growth was inactive. miR-21, microRNA-21.

Although the cytotoxicity of DLN was not investigated in the current study, several studies have already verified the safety of DLN $(34,35)$.

The data of the current study demonstrated that DLN may promote melanoma cell apoptosis and affect cell cycle, thus arresting cells at G1 phase and affecting cell proliferation. Although miR-21 inhibitor also demonstrated anti-cancer effect in melanoma, the co-application of DLN may further promote the effect. miR-21 can regulate multiple signaling pathways and change the expression of tumor-associated protein-coding mRNAs (36). Notably, DLN treatment did not cause a reduction of miR-21, which indicated that the effect of DLN was independent of miR-21. However, additional miR-21 inhibition promoted the anti-cancer effect of DLN.

BCL-2 is a cell-survival factor, while Bax is the most important apoptosis-associated factor. Bax protein can form heteromeric two polymers with BCL-2 (37). The ratio of Bax/BCL-2 is the key factor determining apoptosis (38). P53 can increase the expression of Bax and downregulate the expression of BCL-2 to promote apoptosis (39). P53 can also modulate apoptosis through the death receptor pathway (40). The current study demonstrated that miR-21 inhibitor aggravated the effect of DLN on the expression of BCL-2, Bax and P53. These results suggest that the induced apoptosis may be associated changes in the expression of these genes.

In order to further investigate the effect of DLN and miR-21 inhibitor on tumor growth, a tumor formation assay in nude mice was performed. The results revealed that DLN was more effective in inhibiting tumor development than doxorubicin. Based on previous publications $(12,41,42)$, DLN may have high bioavailability, hydrophobicity and cytotoxicity in tumor cells. miR-21 inhibitor facilitates the anti-cancer activity of DLN in melanoma in vivo as demonstrated by the reduced tumor growth and the morphological changes. However, further experiments are essential for the verification of the mechanisms in vivo.

There were other limitations in this study. The mechanisms associated with the anti-tumor effects of miR-21 inhibitor and DLN require further investigation, although apoptosis may partially explain the effects. Additionally, only one dose of DLN was used in the study. More doses of DLN combined with miR-21 inhibitor should be delivered to further clarify the efficacy. For potential future clinical use, the toxicity of combination of DLN with miR-21 inhibitor in normal cells should also be evaluated.

In conclusion, miR-21 inhibitor promoted the anti-cancer effects of DLN in melanoma cells. Furthermore, the data revealed that the anti-cancer effect of DLN is miR-21-independent, and involves BCL-2, Bax and P53 expression. This study may provide an alternative treatment for melanoma.

\section{Acknowledgements}

Not applicable.

\section{Funding}

This research was supported by the Key $R \& D$ project in Jiangxi Province (grant no. 20161ACG70016).

\section{Availability of data and materials}

The datasets used during the present study are available from the corresponding author upon reasonable request. 


\section{Author's contributions}

$\mathrm{XW}$ and $\mathrm{CZ}$ conceived and designed the experiments; $\mathrm{XW}, \mathrm{XY}$ and $\mathrm{JZ}$ performed the experiments and analyzed the data; XW and $\mathrm{CZ}$ wrote the manuscript. All authors read and approved the final manuscript.

\section{Ethics approval and consent to participate}

All experimental protocols were approved by the Ethics Committee of Ethics Committee of Jiangxi Tumor Hospital (no. 20170312).

\section{Patient consent for publication}

Not applicable.

\section{Competing interests}

The authors declare that they have no competing interests.

\section{References}

1. Gopalani SV, Janitz AE and Campbell JE: Trends in cervical cancer incidence and mortality in Oklahoma and the United States, 1999-2013. Cancer Epidemiol 56: 140-145, 2018.

2. Gunda D, Kido I, Kilonzo S, Nkandala I, Igenge J and Mpondo B: Prevalence and associated factors of incidentally diagnosed prostatic carcinoma among patients Who had transurethral prostatectomy in Tanzania: A retrospective study. Ethiop J Health Sci 28: 11-18, 2018.

3. Lv Y, Song Y, Ni C, Wang S, Chen Z, Shi X, Jiang Q, Cao C and Zuo Y: Overexpression of lymphocyte antigen 6 complex, locus $\mathrm{E}$ in gastric cancer promotes cancer cell growth and metastasis. Cell Physiol Biochem 45: 1219-1229, 2018.

4. Hasan S, Taha R and Omri HE: Current opinions on chemoresistance: An overview. Bioinformation 14: 80-85, 2018

5. Wu X, Xing X, Dowlut D, Zeng Y, Liu J and Liu X: Integrating phosphoproteomics into kinase-targeted cancer therapies in precision medicine. J Proteomics 191: 68-79, 2019.

6. Jindal AB, Bachhav SS and Devarajan PV: In situ hybrid nano drug delivery system (IHN-DDS) of antiretroviral drug for simultaneous targeting to multiple viral reservoirs: An in vivo proof of concept. Int J Pharm 521: 196-203, 2017.

7. Ma X, Gong N, Zhong L, Sun J and Liang XJ: Future of nanotherapeutics: Targeting the cellular sub-organelles. Biomaterials 97: 10-21, 2016.

8. Ying $\mathrm{M}$, Chen $\mathrm{G}$ and $\mathrm{Lu} \mathrm{W}$ : recent advances and strategies in tumor vasculature targeted nano-drug delivery systems. Curr Pharm Des 21: 3066-3075, 2015.

9. Garg T, Bhandari S, Rath G and Goyal AK: Current strategies for targeted delivery of bio-active drug molecules in the treatment of brain tumor. J Drug Target 23: 865-887, 2015.

10. Huang Y, Liu W, Gao F, Fang X and Chen Y: c(RGDyK)-decorated Pluronic micelles for enhanced doxorubicin and paclitaxel delivery to brain glioma. Int J Nanomedicine 11: 1629-1641, 2016.

11. Chen Y, Zhang W, Huang Y, Gao F, Sha X and Fang X: Pluronic-based functional polymeric mixed micelles for co-delivery of doxorubicin and paclitaxel to multidrug resistant tumor. Int J Pharm 488: 44-58, 2015.

12. Yang Z, Luo X, Zhang X, Liu J and Jiang Q: Targeted delivery of 10-hydroxycamptothecin to human breast cancers by cyclic RGD-modified lipid-polymer hybrid nanoparticles. Biomed Mater 8: 025012, 2013.

13. Bohme D and Beck-Sickinger AG: Drug delivery and release systems for targeted tumor therapy. J Pept Sci 21: 186-200, 2015.

14. Ooi CY, Carter DR, Liu B, Mayoh C, Beckers A, Lalwani A, Nagy Z, De Brouwer S, Decaesteker B, Hung TT, et al: Network modeling of microRNA-mRNA interactions in neuroblastoma tumorigenesis identifies miR-204 as a direct inhibitor of MYCN. Cancer Res 78: 3122-3134, 2018.
15. Li M, Zhang F, Su Y, Zhou J and Wang W: Nanoparticles designed to regulate tumor microenvironment for cancer therapy. Life Sci 201: 37-44, 2018.

16. Nogales-Cadenas R, Cai Y, Lin JR, Zhang Q, Zhang W, Montagna $\mathrm{C}$ and Zhang ZD: MicroRNA expression and gene regulation drive breast cancer progression and metastasis in PyMT mice. Breast Cancer Res 18: 75, 2016.

17. Ghosal S, Saha S, Das S, Sen R, Goswami S, Jana SS and Chakrabarti J: miRepress: Modelling gene expression regulation by microRNA with non-conventional binding sites. Sci Rep 6: 22334, 2016.

18. Wandler A, Riber-Hansen R, Hager H, Hamilton-Dutoit SJ, Schmidt $H$, Nielsen BS, Stougaard $M$ and Steiniche T: Quantification of microRNA-21 and microRNA-125b in melanoma tissue. Melanoma Res 27: 417-428, 2017.

19. Hermansen SK, Dahlrot RH, Nielsen BS, Hansen S and Kristensen BW: MiR-21 expression in the tumor cell compartment holds unfavorable prognostic value in gliomas. J Neurooncol 111: 71-81, 2013.

20. Guo L, Ding W and Zheng LM: The C(RgdyK)-conjugated $\mathrm{Fe} 3 \mathrm{O} 4$ nanoparticles with high drug load for dual-targeting integrin alpha(v)beta3-expressing cancer cells. J Nanosci Nanotechnol 14: 4858-4864, 2014.

21. Livak KJ and Schmittgen TD: Analysis of relative gene expression data using real-time quantitative PCR and the 2(-Delta Delta C(T)) method. Methods 25: 402-408, 2001.

22. Yamamoto A: Recent therapeutic strategies for metastatic melanoma: Introduction to invited articles. Int J Clin Oncol, Apr 6 , 2018 (Epub ahead of print).

23. Kim IK, Lane AM, Jain P, Awh C and Gragoudas ES: Ranibizumab for the prevention of radiation complications in patients treated with proton beam irradiation for choroidal melanoma. Trans Am Ophthalmol Soc 114: T2, 2016.

24. Mohamed AA, Richards CJ, Boyle K and Faust G: Severe inflammatory ileitis resulting in ileal perforation in association with combination immune checkpoint blockade for metastatic malignant melanoma. BMJ Case Rep 2018: pii: bcr-2018-224913, 2018.

25. Neto OV, Raymundo S, Franzoi MA, do Carmo Artmann A, Tegner M, Müller VV, Hahn RZ, Alves GV, Schwartsmann G, Linden R and Antunes MV: DPD functional tests in plasma, fresh saliva and dried saliva samples as predictors of 5-fluorouracil exposure and occurrence of drug-related severe toxicity. Clin Biochem 56: 18-25, 2018.

26. Lin C, Zhang X, Chen H, Bian Z, Zhang G, Riaz MK, Tyagi D, Lin G, Zhang Y, Wang J, et al: Dual-ligand modified liposomes provide effective local targeted delivery of lung-cancer drug by antibody and tumor lineage-homing cell-penetrating peptide. Drug Deliv 25: 256-266, 2018.

27. Ke X, Lin W, Li X, Wang H, Xiao X and Guo Z: Synergistic dual-modified liposome improves targeting and therapeutic efficacy of bone metastasis from breast cancer. Drug Deliv 24: 1680-1689, 2017.

28. Belhadj Z, Zhan C, Ying M, Wei X, Xie C, Yan Z and $\mathrm{Lu}$ W: Multifunctional targeted liposomal drug delivery for efficient glioblastoma treatment. Oncotarget 8: 66889-66900, 2017.

29. Zhang HL, Si LB, Zeng A, Long F, Qi Z, Zhao R and Bai M: MicroRNA-21 antisense oligonucleotide improves the sensitivity of A375 human melanoma cell to Cisplatin: An in vitro study. J Cell Biochem 119: 3129-3141, 2018.

30. Lin KY, Chen CM, Lu CY, Cheng CY and Wu YH: Regulation of miR-21 expression in human melanoma via UV-ray-induced melanin pigmentation. Environ Toxicol 32: 2064-2069, 2017.

31. Cifdaloz M, Osterloh L, Graña O, Riveiro-Falkenbach E, Ximénez-Embún P, Muñoz J, Tejedo C, Calvo TG, Karras P, Olmeda D, et al: Systems analysis identifies melanoma-enriched pro-oncogenic networks controlled by the RNA binding protein CELF1. Nat Commun 8: 2249, 2017.

32. Juliano RL, Ming X, Nakagawa $\mathrm{O}, \mathrm{Xu} \mathrm{R}$ and Yoo $\mathrm{H}$ : Integrin targeted delivery of gene therapeutics. Theranostics 1: 211-219, 2011.

33. Jiang X, Sha X, Xin H, Chen L, Gao X, Wang X, Law K, Gu J, Chen Y, Jiang Y, et al: Self-aggregated pegylated poly (trimethylene carbonate) nanoparticles decorated with c(RGDyK) peptide for targeted paclitaxel delivery to integrin-rich tumors. Biomaterials 32: 9457-9469, 2011.

34. Qiu L, Hu Q, Cheng L, Li L, Tian C, Chen W, Chen Q, Hu W, Xu L, Yang J, et al: cRGDyK modified $\mathrm{pH}$ responsive nanoparticles for specific intracellular delivery of doxorubicin. Acta Biomater 30: 285-298, 2016. 
35. Chen Y, Zhang W, Huang Y, Gao F and Fang X: In vivo biodistribution and anti-tumor efficacy evaluation of doxorubicin and paclitaxel-loaded pluronic micelles decorated with $\mathrm{c}(\mathrm{RGDyK})$ peptide. PLoS One 11: e0149952, 2016.

36. Ribas J and Lupold SE: The transcriptional regulation of miR-21, its multiple transcripts, and their implication in prostate cancer. Cell Cycle 9: 923-929, 2010.

37. Wang Q, Zhang L, Yuan X, Ou Y, Zhu X, Cheng Z, Zhang P, Wu X, Meng Y and Zhang L: The relationship between the $\mathrm{Bcl}-2 / \mathrm{Bax}$ proteins and the mitochondria-mediated apoptosis pathway in the differentiation of adipose-derived stromal cells into neurons. PLoS One 11: e0163327, 2016.

38. Fan Y, Yang F, Cao X, Chen C, Zhang X, Zhang X, Lin W, Wang X and Liang C: Gab1 regulates SDF-1-induced progression via inhibition of apoptosis pathway induced by PI3K/AKT/Bcl-2/BAX pathway in human chondrosarcoma. Tumour Biol 37: 1141-1149, 2016.
39. Ou X, Lu Y, Liao L, Li D, Liu L, Liu H and Xu H: Nitidine chloride induces apoptosis in human hepatocellular carcinoma cells through a pathway involving p53, p21, Bax and Bcl-2. Oncol Rep 33: 1264-1274, 2015.

40. Dolka I, Król M and Sapierzyński R: Evaluation of apoptosis-associated protein (Bcl-2, Bax, cleaved caspase-3 and p53) expression in canine mammary tumors: An immunohistochemical and prognostic study. Res Vet Sci 105: 124-133, 2016.

41. Li C, Shen J, Wei X, Xie C and Lu W: Targeted delivery of a novel palmitylated D-peptide for antiglioblastoma molecular therapy. J Drug Target 20: 264-271, 2012.

42. Li G, Song YZ, Huang ZJ, Chen K, Chen DW and Deng YH: Novel, nano-sized, liposome-encapsulated polyamidoamine dendrimer derivatives facilitate tumour targeting by overcoming the polyethylene glycol dilemma and integrin saturation obstacle. J Drug Target 25: 734-746, 2017. 